This is the post-print version of the following article:

Lucas-Estañ MC, Gozalvez J, Sanchez-Soriano J. Bankruptcy-based Radio Resource Management for Multimedia Mobile Networks. European Transactions on Telecommunications, Volume 23, Issue 2, pages 186-201, March 2012 | DOI: 10.1002/ett.1525

which has been published in final form at http://onlinelibrary.wiley.com/doi/10.1002/ett.1525/abstract.

\title{
Bankruptcy-based Radio Resource Management for Multimedia Mobile Networks
}

\author{
M.C. Lucas-Estañ ${ }^{\#}$, J. Gozalvez ${ }^{\#}$ J. Sanchez-Soriano* \\ \# Ubiquitous Wireless Communications Research (Uwicore) Laboratory, University Miguel Hernandez of Elche, Spain \\ * Operations Research Center, University Miguel Hernandez of Elche, Spain, \\ m.lucas@umh.es, j.gozalvez@umh.es, joaquin@umh.es
}

\begin{abstract}
The transmission of bandwidth demanding multimedia applications in capacity constrained mobile radio networks requires optimizing the usage and assignment of radio resources following the varying Quality of Service (QoS) requirements characteristics of multimedia traffic environments. Considering the capacity of bankruptcy theory to deal with situations where the demand for resources is higher than its availability, this work proposes the application of bankruptcy theories to design efficient radio resource management policies that provide the highest possible QoS levels while guaranteeing user fairness.
\end{abstract}

Index Terms - Bankruptcy theory, mobile radio networks, multimedia traffic, Radio Resource Management.

\section{INTRODUCTION}

The increasing introduction and demand for multimedia services in mobile networks is urging operators to design Radio Resource Management (RRM) policies that efficiently use the scarce available radio resources. The simultaneous allocation of multiple radio resources to a given user can increase its throughput and reduce its end-to-end delay. However, considering the limited number of radio resources, such improvements could be produced at the cost of blocking new users, or unfairly assigning radio resources among users. In this context, it is important to design RRM policies that avoid channel stagnation while considering the system load and the user Quality of Service (QoS) requirements. It is also important to note that different traffic services might have varying QoS requirements, and such variability could be exploited to design efficient and fair RRM mechanisms in multimedia traffic scenarios.

To design adequate radio resource distribution mechanisms, several factors should be taken into account. First of all, their computational cost should be feasible to allow for their implementation. The work reported in [1] showed that assignment schemes considering all possible distribution patterns result in excessive computational costs that currently prevent their use in mobile communication systems. Radio resource distribution policies should also be able to assign the adequate number of resources to each user based on their QoS demand and the system load. To this end, the work reported in [2] highlighted the performance improvement in terms of admission probability and dropping probabilities achieved by Admission Control, Packet Scheduling and Congestion Control mechanisms based on QoS differentiation.

User fairness is also becoming an important objective in the design of RRM techniques. A scheduling algorithm designed to balance the requirements of users and service providers is reported in [3]. The authors proposed utility functions that try to ensure a fair distribution of radio resources among users, where user fairness is defined in terms of the distribution of average throughput per user. In [4], the resource allocation 
problem in a TD-CDMA system is modelled as a nonlinear optimization problem with the aim of achieving a trade-off between throughput maximization and fairness. The proposed algorithm maximizes the sum of normalized rates instead of throughput in order to increase the fairness in the rate allocations while maintaining throughput at a high value. The resource allocation algorithm is applied considering only non-real time users and the radio resources that remain unassigned after guaranteeing the QoS requirements of real-time users. An interesting study addressing the concept of user fairness is also found in [5], where the authors propose a fair resource allocation approach for interference-limited communication systems based on game theory and bargaining problems. The proposed scheme is shown to achieve the sought compromise between fairness and efficiency. While the reviewed contributions aimed at achieving equal radio resource or throughput assignments, the RRM techniques proposed in this work have been designed to provide homogenous QoS levels to users in multimedia environments, where different traffic or service classes might have varying QoS requirements.

Other studies that also considered the use of utilitybased radio resource distribution policies were suggested in [6] and [7]. For example, [6] proposes a RRM technique for CDMA uplink transmissions that bases its decisions on aspects related to network resources and user preferences (e.g. data rate or signal quality). The study is focused on maximizing the total network revenue in order to achieve an optimal transmission rate for each user and guarantee acceptable signal quality levels. The study reported in [7] proposes the use of economic concepts to address the radio resource assignment problem by defining Vickrey auctions derived to satisfy user QoS requirements for constant bit rate services. To this aim, the study defines utility functions based on the price users are willing to pay for radio resources. Users participate in the auctions with their utility functions, and an auction is made for each available resource. The proposed mechanism resulted in that users achieved very high satisfaction levels, or were not assigned any resources. Of particular interest is also the study reported in [8] that proposed a radio resource assignment scheme that seeks to fairly distribute available resources. To establish their distribution policies, the authors first estimate the bandwidth needed by a user to guarantee an adequate transmission. Based on such estimates, the reported scheme equally distributes the available bandwidth among users. If all users are satisfied, and there is yet available bandwidth, the available resources are again equally distributed among users. It is important to note that this proposal distributes frequency bands, which is a resource considered continuous and infinitively divisible. On the other hand, this work considers radio resources (timeslots or codes) of discrete nature, and the proposed resource distribution policies are aimed at fairly providing homogeneous QoS levels for all active users.

This paper proposes to further expand the use of economics concepts to address the radio resource assignment problem in multimedia traffic scenarios by developing innovative policies using bankruptcy theory. In bankruptcy situations, the value of a company is inferior to the sum of its debts, and adequate distribution policies are needed to divide its net value among its creditors [9]. Given the similarity of the problems dealt with by bankruptcy and the radio resource distribution dilemma in highly loaded multimedia traffic environments, this work extends the work reported in [10] and [11], and analyses innovative bankruptcy-based radio resource assignment proposals designed to maximise the resources' efficiency and user satisfaction fairness, while exploiting the varying QoS demands from different service classes in multimedia networks. To this aim, the bankruptcy distribution rules have been designed to work with discrete resources, and to utilize utility functions that are not directly proportional to the number of resources allocated to a user. The performance and operation of the proposed bankruptcybased RRM techniques, including their computational cost, is compared under different operating conditions to other RRM schemes, in particular the Maximum Utility Increase (MAUI) and Required dATa ratE (RATE) techniques.

\section{RAdio Resource AssignMent Proposals}

\subsection{Traffic Service Utility Functions}

Utility functions try to characterize the QoS satisfaction level experienced by a user based on the requested traffic service and the radio resources it has been assigned (combination of RAT and number of radio resources assigned within that RAT). This is a challenging task because user satisfaction is a subjective concept that heavily depends on user perceptions. The defined utility functions try to express the perceived user QoS as the link quality, and therefore data rate, varies. To establish the utility functions, the minimum, mean, and maximum QoS levels demanded by users are first defined per service class as illustrated in Fig. 1. This work considers a multimedia traffic scenario with email 

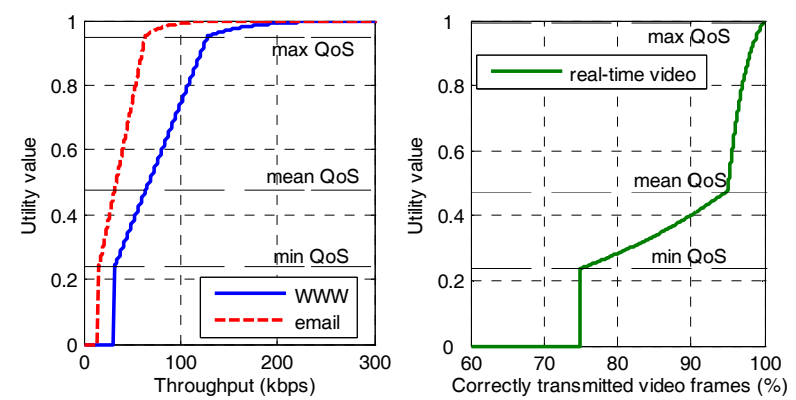

Fig.1. Utility functions per traffic service.

(background), web (interactive) and real-time H.263 video (at 16, 32 and 64kbps mean bit rates) users.

For web and email services, utility values are expressed in terms of the user throughput. The minimum, mean and maximum QoS levels for web users have been defined as the throughput needed to satisfactorily transmit $90 \%, 95 \%$ and $97.5 \%$ of web pages in less than 4 seconds as established by the 3GPP TS 22.105 recommendations. These high percentiles have been selected due the high transmission reliability requirements of non-real time data services. Web traffic is here modeled using the work reported in [12], whereas [13] has been used to model email traffic. The email model considers the transmission of emails with and without attachments, which makes it difficult to successfully transmit emails with large attachments within the 4 seconds $3 \mathrm{GPP}$ recommendations. Consequently, the email QoS thresholds have been established based on the throughput required to satisfactorily transmit $65 \%, 75 \%$ and $80 \%$ of the emails (with or without attachments). Once the QoS satisfaction thresholds have been established for web and email services, the utility functions have been defined so that users perceive a null utility value if their minimum QoS demand is not satisfied. This condition avoids assigning radio resources to users that would experience very poor QoS levels. Web and email user satisfaction linearly grows with the experienced throughput between the minimum and maximum QoS thresholds. Utility values equal to one have been avoided for web and email transmissions to account for the transmission reliability requirements of these services, and the dependence of the achievable throughput levels on the experienced channel quality conditions.

For real-time video services, video frames are considered to be satisfactorily transmitted if they are transmitted before the next video frame is to be transmitted. Consequently, the utility functions for realtime video services have been defined based on the percentage of correctly transmitted video frames, and the real-time video utility functions are independent of the mean video bit rates. The real-time video QoS satisfaction thresholds have been established considering the H.263 traffic model described in [14] and the indications provided in [15]. The studies reported in [15] show that a $25 \%$, or even higher, dropping rate does not have a catastrophic effect on the QoS perceived by H.263 video users, and that dropping rates as high as 5\% can be overcome if appropriate transmission techniques are invoked. Based on these results, the minimum and mean QoS satisfaction levels correspond to guaranteeing that $75 \%$ and $95 \%$ of video frames are transmitted before the next video frame needs to be transmitted. The maximum utility value for real-time video users has been set equal to one, and is achieved when all video frames are transmitted before the next video frame is to be transmitted. Although the $5 \%$ difference between the mean and maximum QoS levels might look negligible, this $5 \%$ includes the H.263 I-frames. These frames include information of independently coded images in a video sequence, and are also used to code/decode other images exploiting temporal redundancy. As a result, Iframes have a significant impact on the user perceived QoS level, and require high transmission rates due to their potential large size. Similarly to web and email users, real-time video users also perceive a null utility value below the minimum QoS threshold. Following the indications in [15] that highlight that an acceptable video quality requires a high percentage of correctly received video frames, the video utility increases slowly with the percentage of transmitted frames until the mean QoS level is achieved, and then rapidly until the maximum QoS level

The work reported in this paper considers the GERAN (GSM/EDGE Radio Access Network) radio interface (3GPP TS 43.064) as a TDMA representative technology over which to test the bankruptcy-based RRM proposals. Of course, the proposed techniques can be used and adapted to other standards and multiple access technologies requiring the definition of policies to distribute multiple radio resources among active users. However, this work is focused on the demonstration of the benefits that can be achieved through the use of bankruptcy-based techniques in radio resource management problems, and the adaptation to other particular systems, although possible, is out of the scope of the paper. In GERAN, a radio resource corresponds to a TDMA timeslot (TS). GERAN allows the allocation of multiple radio resources to a single user, with the maximum number of resources that can be assigned being equal to eight timeslots. GERAN also implements an adaptive radio interface that dynamically varies the 
used transmission mode (modulation and coding scheme, MCS) according to the experienced channel quality conditions. Such adaptive operation and the continuously varying channel quality conditions difficult the possibility to predict the mean bit rate that could be achieved with a given number of radio resources. To relate the utility functions to the number of assigned radio resources, this work considers the MCS5 transmission mode, which provides a mean bit rate of $22.4 \mathrm{kbps}$ per timeslot. This transmission mode has been identified as the most widely used in system level simulations conducted based on the scenario reported in Section 3. Different system configurations would require identifying again the transmission mode that is used more often, and consequently redefining the utility values, although this would not influence the obtained performance trends. With the selection of a transmission rate per timeslot, the relation between assigned radio resources and utility values can be directly obtained using Fig. 1 for email and web services, and is depicted in Fig. 2. For real-time H.263 video services, an additional step is necessary. A cumulative distribution function (CDF) of the throughput needed to transmit each video frame before the next video frame is to be transmitted is derived following the implemented H.263 video model [14]. Through these CDFs, the percentage of video frames reported in Fig. 1 can be related to the corresponding necessary throughputs for the various video bit rates considered in this work $(16,32$ and $64 \mathrm{kbps}$ ), and consequently to the needed radio resources once the MCS5 transmission mode has been selected to estimate the throughput achievable per radio resource.

The final discrete utility values $\left(U_{s}: \mathrm{N} \rightarrow[0,1]\right.$, where $\mathrm{N}$ is the set of all non negative integer numbers) defined per traffic service $(s)$ and number of assigned radio resources to a user $(r \in \mathrm{N})$ are shown in Fig. 2. These utility functions satisfy that $U_{s}(0)=0$ and $U_{s}(r) \leq U_{s}(r+1)$. It is important to note that the minimum number of radio resources needed to achieve a positive utility value

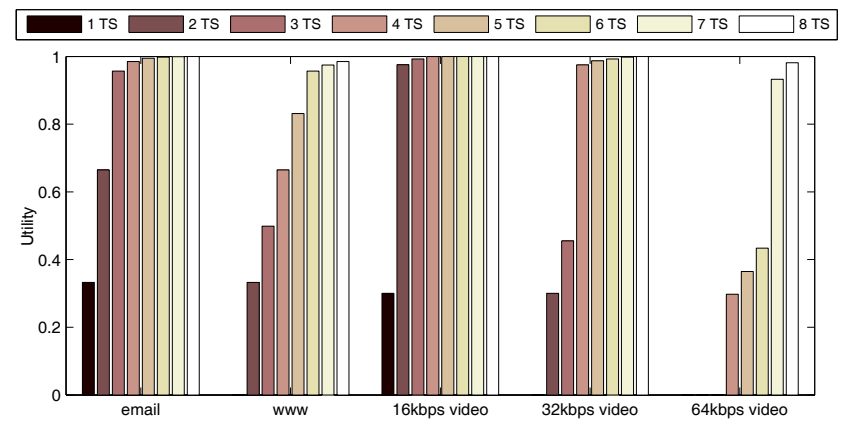

Fig.2. Utility values per traffic service and number of radio resources. $\left(l_{i}=\min \left\{r \in \mathrm{N}: U_{s_{i}}(r)>0\right\}\right.$ for user $i$ demanding service $\left.s_{i}\right)$ is higher than one for certain traffic services. This is due to the defined QoS levels depicted in Fig. 1, and the fact that the implemented policies try to guarantee, at least, the minimum QoS level for each user. As a result, each service class requires a different number of radio resources to obtain a utility value greater than zero. Furthermore, the discrete utility functions satisfy that if $0<U_{s}(r)<1, U_{s}(r)<U_{s}(r+1)$. This property means that when a user begins to perceive positive satisfaction, then every additional resource leads to a strictly positive satisfactory increment, except when its maximum QoS demand or utility value is achieved. It is important to note that although different utility values would be needed if modifying the system operating conditions, the multimedia traffic scenario or the employed radio interface, the proposed methodology to establish the utility functions would still be valid. In addition, the comparison of the bankruptcy based techniques with the RATE (Required dATa rate) mechanism (defined in Section 2.4) will highlight the adequate definition of the utility functions.

\subsection{Bankruptcy-based Radio Resource Assignment}

Bankruptcy theory has been proposed to solve situations where the number of claimed resources exceeds those available. In these cases, policies are defined to decide how scarce resources should be fairly distributed among the claiming creditors. Due to the high similarity with the radio resource assignment dilemma under high user load scenarios, this paper evaluates the potential of bankruptcy theory to define efficient and fair radio resource assignment policies. In particular, this paper evaluates the potential of bankruptcy theory through the adaptation of two bankruptcy rules, Constraint Equal Award (CEA) and Constraint Equal Loss (CEL) [9], which were originally proposed to achieve fair distributions of scarce resources. For the formal definition of a bankruptcy situation, let's consider a value $E \in \mathrm{R}_{+}$that has to be divided among $N$ agents with claims adding up to more than $E$. For each $i \in N, c_{i} \in \mathrm{R}_{+}$represents the resources requested by $i$, and $c \equiv\left(c_{i}\right)_{i \in N}$ the requests vector. In this context, a resources distribution dilemma is denoted by $(c, E) \in \mathrm{R}_{+} \times \mathrm{R}_{+}$such that $\Sigma c_{i} \geq E$.

Traditionally, bankruptcy theory has been applied to the distribution of continuous resources. However, the definition of bankruptcy-based radio resource assignment policies for mobile radio networks should work with radio resources of discrete nature. It is 
important to note that, considering the development of bankruptcy theory over the years, only recently has been addressed the extension of bankruptcy distribution rules to handle discrete resources. An example is the work reported in [16], which is based on utility values that are directly proportional to the number of assigned resources (as in the original bankruptcy rules). On the other hand, the resource distribution techniques proposed in this work are designed considering utility values that are not directly proportional to the number of assigned radio resources (Section 2.1). To the author's best knowledge, this is the first work that applies bankruptcy rules to solve RRM problems, and considers the extension of bankruptcy policies to distribute discrete resources considering a variety of user profiles and requests. This working condition provides a better representation of multimedia wireless scenarios, where users from different service classes might experience unequal QoS levels when receiving the same number of radio resources.

\subsubsection{General Framework}

In mobile radio networks with a finite number of available radio resources, $R$, a finite set of users, $N$, claim for different services ( $s \in S$, where $S$ is the set of all traffic services offered in the system). Each traffic service can be characterized by varying QoS requirements and resource demands and, in general, the QoS perceived by users requesting different services is not directly proportional to the number of assigned resources. This work proposes the use of the utility functions defined in Section $2.1\left(U_{s}: \mathrm{N} \rightarrow[0,1]\right)$ to quantify the radio resource requirement of each service class to achieve different satisfaction levels.

One of the objectives of the proposed bankruptcybased policies is user fairness. Achieving this objective can be challenging due to the discrete nature of radio resources, and the potentially high number of users in a system. In this context, a user priority criterion, represented by $<_{L}\left(i<_{L} j\right.$ indicates that user $i$ is higher priority than user $j$ ), has been established. In this work, $<_{L}$ defines that users will be served based on the following service priorities: 64kbps video (highest priority), 32kbps video, $16 \mathrm{kbps}$ video, web and email (lowest priority). When two users are demanding the same type of service, other information can be taken into account for assigning resources; in this work, the higher the waiting time, the higher priority is assigned to the user by $<_{L}$. The bankruptcy-based policies proposed in this work also seek to provide users with an adequate and maximum possible QoS level. Accordingly, the radio resource demands of each user $i$, represented by $c_{i}$, can be established as the minimum number of resources needed to achieve the maximum utility value when the user is demanding service $s_{i}$. Mathematically, $c_{i}$ is expressed as $c_{i}=\min \operatorname{argmax}\left\{U_{s_{i}}(r): r \in \mathbf{N}, r \leq R\right\}$. In high user load scenarios, it could be possible that $\Sigma c_{i} \geq R$, and even that $\Sigma l_{i} \geq R$ ( $l_{i}$ denotes the minimum number of radio resources needed to achieve a positive utility value as defined in Section 2.1).

\subsubsection{Discrete CEA for Mobile radio resource distribution (DCEAM)}

The CEA distribution rule assigns an equal number of resources to all users, subject to none receiving more than its claim. The definition of the CEA distribution rule can then be established as follows [9]:

$$
C E A(c, E) \equiv\left(\min \left\{c_{i}, \lambda\right\}\right)_{i \in N}, \text { where } \sum_{i} \min \left\{c_{i}, \lambda\right\}=E
$$

In (1), $\lambda$ is chosen so that all users receive an equal number of resources. CEA equally divides the value $E$ among the $N$ agents participating in the distribution process $(E / N)$. If the amount received by an agent is greater than its claim, that agent only receives the resources corresponding to its claim, and the remaining resources are equally divided among the rest of agents. This process must be done for all agents. Consequently, the users with the lowest resource demands achieve a higher satisfaction than those requiring more resources.

To tackle the distribution of discrete resources, the authors propose the Discrete CEA for Mobile radio resources distribution (DCEAM) mechanism. DCEAM follows the CEA distribution principles, but has been designed to distribute resources of discrete nature (for example, timeslots for TDMA systems or codes for CDMA). The DCEAM proposal does not aim to equally distribute resources among users, but rather equally distribute user satisfaction levels. In addition, an important novelty of the DCEAM proposal is that it is based on utility values that are not directly proportional to the number of assigned radio resources. As a result, to achieve an equal satisfaction level, DCEAM might result in an unequal distribution of resources without affecting the user-perceived QoS. To achieve its objectives, the DCEAM operation is based on the following rules:

- All users begin the distribution process without assigned resources ${ }^{1}$.

\footnotetext{
${ }^{1}$ For simplicity, it has been assumed that all users begin the distribution round without assigned resources. However, real-time users can maintain some previously assigned resources as explained in the following paragraph.
} 
- $x_{i}^{0}=0$ for all $i \in N$, where $x_{i}^{k}$ is the number of resources assigned to user $i$ when $k$ resources $(k \in$ $[1, R])$ have been distributed.

- Radio resources are assigned one by one $(R$ iterations).

- Each resource is assigned to the user with the lowest utility value to try to achieve equal user utility values: - $\mathrm{U}^{k}=\left(\mathrm{U}^{1, k}, \mathrm{U}^{2, k}, \ldots, \mathrm{U}^{n, k}\right)$ where $\mathrm{U}^{i, k}=U_{s_{i}}\left(x_{i}^{k}\right)$ for all $i \in N$.

- $N^{k}=\operatorname{argmin}\left\{\mathrm{U}^{i, k}: i \in N\right\}-\left\{i \in N: x_{i}=c_{i}\right\}$, where $N^{k}$ is the set of users with the lowest utility value.

- When different users have the same utility value, users are served based on the priority criterion $<_{L}$.

- User $h \in N^{k}$ is chosen such that $h<_{L} j$ for all $j \in N^{k}$.

It is important to note that this work assumes that each time a user requests access to the system or a user ends its transmission, the distribution proposal is applied and all radio resources are re-distributed ${ }^{2}$. Only active realtime users can maintain the amount of previously assigned radio resources guaranteeing their minimum QoS level, and will compete with the rest of users for additional resources. If $x_{i}^{-1}$ denotes the number of resources assigned to an active video user $i$ in the previous distribution round, this condition can be mathematically expressed as follows: if $x_{i}^{-1} \geq l_{i}$ then $x_{i}^{0}=$ $l_{i}$, otherwise $x_{i}^{0}=x_{i}^{-1}$. On the other hand, non real-time users are not guaranteed to maintain their previously assigned radio resources; $x_{i}^{0}=0$ for a non-real-time user $i$ since they have to compete for any radio resource at each distribution round.

\subsubsection{Discrete CEL for Mobile radio resource distribution (DCELM)}

An alternative to CEA is the CEL rule, where the distribution process focuses on the losses claimants incur as opposed to what they receive. Consequently, the CEL technique distributes the resources to achieve equal losses for all agents participating in the distribution process, which can then be expressed as:

$$
C E L(c, E) \equiv\left(\max \left\{0, c_{i}-\lambda\right\}\right)_{i \in N} \text {, where } \sum_{i} \max \left\{0, c_{i}-\lambda\right\}=E
$$

In (2), $\lambda$ is chosen so that users suffer equal resource losses. CEL considers that initially each agent has been assigned its maximum resources request. Given that such requests cannot be fulfilled in loaded systems, CEL equally reduces the amount of resources to each agent. CEL adds for all users the amount of resources requested per user to achieve maximum user satisfaction, and computes the difference between this value and the amount of total available resources $\sum c_{i}-E$. The resulting value is equally divided between the $N$ agents participating in the distribution process $\left(\sum c_{i}-E\right) / N$, and the result represents the number of resources that each agent will loose given its initial maximum assignments. If this amount is greater than an agent's claim, that agent does not receive any resources and the different $c_{k}-\left(\sum c_{i}-E\right) / N$ resources are equally unassigned to the rest of agents. When the CEL distribution rule is applied, agents with the highest demands achieve higher satisfaction levels [9].

Similarly to DCEAM, this work also evaluates DCELM (Discrete CEL for Mobile radio resources distribution), an adaptation of the CEL distribution rule to the radio resource assignment dilemma working with discrete resources. As it was the case for DCEAM, DCELM bases its operation on equal user satisfaction losses and not equal radio resources losses. To exploit the varying QoS requirements of different traffic services, the DCELM proposal also considers the utility values defined in Section 2.1. The DCELM mechanism operates as follows:

- Users receive their maximum resources requests at the beginning of a distribution round.

- $x_{i}^{0}=c_{i}$ for all $i \in N$.

- Radio resources are unassigned one by one until the total number of assigned radio resources equals the number of available radio resources $\left(\sum x_{i}=R\right)$.

- Each resource is removed from the user with the lowest utility losses:

- $\mathrm{U}^{k}=\left(\mathrm{U}^{1, k}, \mathrm{U}^{2, k}, \ldots, \mathrm{U}^{n, k}\right)$ where $\mathrm{U}^{i, k}=U_{s_{i}}\left(x_{i}^{k}\right)$ for all $i \in N$.

- $N^{k}=\operatorname{argmin}\left\{\left(1-\mathrm{U}^{i, k}\right): i \in N\right\}=\operatorname{argmax}\left\{\mathrm{U}^{i, k}: i \in N\right\}$, where $N^{k}$ is the set of users with the lowest utility losses.

-When different users experience the same utility loss, radio resources are first removed from the user with lower priority.

- User $h \in N^{k}$ such that $j<_{L} h$ for all $j \in N^{k}$ is chosen.

Given that each service class has different resources needs, DCELM does not equally unassign resources to each user, but unassigns resources to achieve equal utility losses. As a result, the number of finally assigned resources per user might be different for each service class.

\footnotetext{
2 A different assumption would produce different system performance numerical values, but would not influence the bankruptcy-based RRM performance trends and the comparative study outcome.
} 


\subsection{MAximum Utility Increase (MAUI)}

Following the philosophy of the proposals reported in [6] and [7] that aim at maximizing the total system utility value to increase the system's revenue, the MAUI technique is aimed at maximizing the global utility value that could be achieved with the available resources. To achieve its objective, MAUI is based on the following rules:

- All users begin the distribution process without assigned resources (except active real-time video users following the previously defined rule).

- $x_{i}^{0}=0$ for all $i \in N$.

- Radio resources are assigned one by one $(R$ iterations).

- Each resource is assigned to the user that will experience the higher utility increase when assigned such radio resource:

- $\mathrm{I}^{k}=\left(\mathrm{I}^{1, k}, \mathrm{I}^{2, k}, \ldots, \mathrm{I}^{n, k}\right)$ where $\mathrm{I}^{i, k}=U_{s_{i}}\left(x_{i}^{k}+1\right)-U_{s_{i}}\left(x_{i}^{k}\right)$ for all $i \in N$.

- $N^{k}=\operatorname{argmax}\left\{\mathrm{I}^{i, k}: i \in N\right\}-\left\{i \in N: x_{i}=c_{i}\right\}$, where $N^{k}$ is the set of users with the highest utility increase.

- When different users experience the same utility increase, users are served based on the priority criterion $<_{L}$.

- User $h \in N^{k}$ is chosen such that $h<_{L} j$ for all $j \in N^{k}$.

Since MAUI sequentially assigns each radio resource evaluating the utility increase it independently produces, the final achieved global utility value might not be the highest possible one compared to the case in which all assignment combinations are computed. However, it provides a sub-optimal solution with a reduced computational cost that allows for its real implementation compared to proposals aimed at evaluating all possible resource distributions [1].

\subsection{Required dATa ratE (RATE)}

Contrary to previous proposals, RATE does not base its decision criterion on utility functions. Instead, and following the philosophy of proposals reported in e.g. [3] and [4], RATE bases its decision criterion on the estimated transmission data rate needed by each user to satisfactorily end its active transmission. RATE estimates the transmission data rate required by each user following the $3 \mathrm{GPP}$ and real-time QoS requirements defined in Section 2.1. Subsequently, the RATE scheme assigns the number of radio resources necessary to achieve the identified data rates. In this context, the target data rates for each service class are calculated as follows:

- For real-time video users, the required data rate is defined as a function of the amount of data pending to be transmitted (DataToTransmit) and the difference between the time at which the next frame is to be transmitted (MaxTime) and the current time (CurrentTime):

$$
r_{\text {video }}=\frac{\text { DataToTransmit }}{\text { MaxTime }- \text { CurrentTime }}
$$

- For email users, the required data rate is computed as the ratio between the amount of data pending to be transmitted and the difference between the maximum time to satisfactorily transmit the email (4 seconds following the 3GPP recommendations) and the time that has been spent since the transmission started (SpentTime):

$$
r_{\text {email }}=\frac{\text { DataToTransmit }}{4-\text { SpentTime }}
$$

- Since a web page is composed of several objects, the target data rate should be calculated to be satisfactory to a large part of these individual objects. Following the implemented web traffic model (section 3), 99\% of web pages are composed by 7 or less objects. In addition, the inactivity time between these objects for $99 \%$ of web pages does not exceed 1.362 seconds. In this case, the time to satisfactorily transmit a web object $\left(t_{o b j}\right)$, and the target data rate for a web page have been estimated as follows:

$$
\begin{aligned}
t_{o b j} & =\frac{4 s-1.362 s}{7 \text { objects }}=0.377 \mathrm{~s} \\
r_{\text {web }} & =\frac{\text { DataToTransmit }}{t_{\text {obj }}-\text { SpentTime }}
\end{aligned}
$$

Contrary to real-time services, web and email users do not abort their transmissions if they exceed the maximum time established to be considered satisfactory. For web and email transmissions that continue beyond the 4 seconds mark, the RATE scheme considers as target data rate a fixed rate corresponding to the minimum QoS levels defined in Section 2.1 (22.4kbps* $l_{i}$ considering the MCS5 transmission mode). Once the required data rates are established, the RATE operation is based on the following rules:

- All users begin the distribution process without assigned resources (except active real-time video users following the previously defined rule).

- $x_{i}^{0}=0$ for all $i \in N$.

- Users are classified per service class, and the different sets of users are served following the identified service priority order (section 2.2.1.

-Within each set of users demanding the same service class, the DCEAM rule is applied. In this case, the 
user requests correspond to the computed data rates, and not to the resource demands as in the original DCEAM policy:

- Each resource is assigned to the user with the lowest assigned data rate to try to achieve equal data rates:

- $\mathrm{R}^{k}=\left(\mathrm{R}^{1, k}, \mathrm{R}^{2, k}, \ldots, \mathrm{R}^{n, k}\right)$ where $\mathrm{R}^{i, k}=22.4^{*} x_{i}^{k}$ for all $i \in N$ (the MCS5 transmission mode is considered).

- $N^{k}=\operatorname{argmin}\left\{\mathrm{R}^{i, k}: i \in N\right\}-\left\{i \in N: \mathrm{R}^{i, k} \geq r_{i}\right\}$, where $N^{k}$ is the set of users with the lowest data rates, and $r_{i}$ is the target data rate for user $i$.

- When different users request the same data rate, the radio resource is assigned to the user experiencing a higher waiting time:

- User $h \in N^{k}$ is chosen such that $h<_{L} j$ for all $j \in N^{k}$.

- When a set of users demanding the same traffic service is satisfied and some radio resources are still unassigned, the set of users demanding the lower priority traffic service is then served.

\section{SPHERE SIMULATION PLATFORM}

The performance of the proposed radio resource assignment policies has been assessed using the SPHERE (Simulation Platform for HEterogeneous wiREless systems) platform, an advanced system level and event-driven downlink simulation platform developed at the University Miguel Hernandez of Elche [17]. The SPHERE platform accurately models the

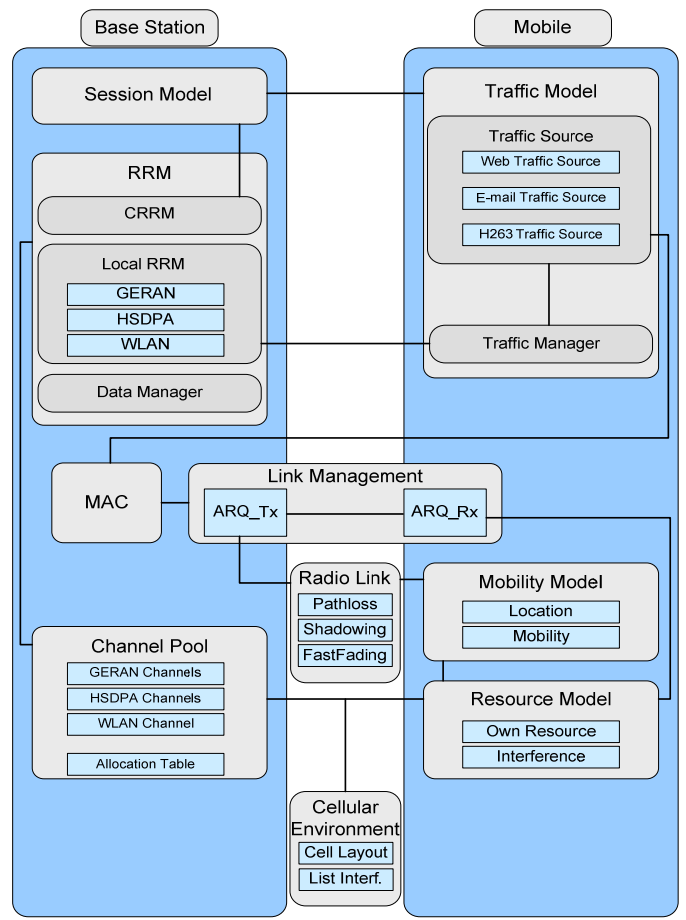

Fig.3. SPHERE logical structure.
GERAN radio interface (3GPP TS 43.064) and implements RRM features required to conduct research studies on the design, evaluation and optimization of RRM techniques. The logical structure of the SPHERE simulation platform is shown in Fig. 3. The modular and scalable design of SPHERE guarantees an easy adaptation of the platform's configuration to specific requirements, and allows the rapid integration of new features.

The SPHERE platform models the GERAN radio interface at the timeslot level. SPHERE implements all GERAN transmission modes, and models its adaptive radio interface through the use of Link Adaptation (LA). This technique periodically selects the optimum transmission mode for the experienced channel quality conditions. For non real-time services, the transmission mode that maximises the throughput is selected. For real-time services, the algorithm proposed in [18] has been used. The LA updating period has been set to $100 \mathrm{~ms}$, except for the start of any new transmission for which an initial LA updating period of $60 \mathrm{~ms}$ has been selected. Erroneously received non real-time data is retransmitted by means of a selective-repeat ARQ protocol following the specifications reported in (3GPP TS 44.060); retransmission protocols are not applied for delay-sensitive real-time services.

SPHERE emulates email, web and H.263 real-time video transmissions [17] at the packet level, which allows an accurate evaluation of the final user perceived QoS. Each traffic type is simulated at the session level, with the session arrival modelled through a Poisson process, and the session duration modelled by Poisson, geometric and exponential distributions for email, web and H.263 users, respectively. Regarding the propagation model, SPHERE models the pathloss by means of the Okumura-Hata COST 231 model, and the shadowing through a lognormal distribution with $6 \mathrm{~dB}$ standard deviation. To reduce the complexity of system level simulations, the physical layer effects resulting from the probabilistic nature of the radio environment have been included by means of Look-Up Tables, extracted from link level simulations and mapping the Packet Error Rate to the experienced channel quality.

The implemented simulation scenario represents a cellular network with 25 cells of $1 \mathrm{~km}$ radio, a cluster size equal to 4 , and $120^{\circ}$ sectorized antennas. The reference scenario considers 16 active users per cell: 5 email and web users, and 6 users demanding real-time video services. Real-time video users are equally distributed among the three simulated video mean bit rates $(16,32$ and $64 \mathrm{kbps})$. Other cell loads have also been simulated to analyse the efficiency of the proposed RRM techniques as the system load varies; these loads are specified in Section 4 where the performance of the 
RRM proposals is evaluated. Users move at an average speed of $50 \mathrm{~km} / \mathrm{h}$ based on a macro-cellular mobility model, although they are bounded to a specific cell. A single frequency carrier ( 8 timeslots) per cell has been considered.

\section{Performance Evaluation}

The user satisfaction is used in this paper as the main performance metric. It accounts for the percentage of web pages or emails transmitted in less than 4 seconds, and the percentage of video frames transmitted before the next frame is to be transmitted. To analyse the potential of the radio resource assignment proposals, the performance obtained with a static radio resource assignment policy is also presented. This policy assigns each user the number of resources requested, and if there are not enough resources available, the user is queued. The static radio resource policy has been implemented considering that the demand for resources is equal to the minimum, mean and maximum user QoS levels defined in Section 2.1. As shown in Table I, the static radio resource assignment policy is not able to obtain suitable QoS satisfaction levels for the most demanding services. The user satisfaction levels achieved with the proposed policies are depicted in Table II. The obtained results show that all the proposals generally achieve higher user satisfaction levels for the services with the more restrictive delay requirements (real-time video services), which is reasonable since real-time video users are served with a highest priority. In any case, it is important to emphasize that the achieved user satisfaction levels are highly influenced by the number of available radio resources and the emulated system load. Although the performance of the more poorly served users will be significantly improved under lower system loads, this work is aimed at proposing, evaluating and optimising radio resource assignment policies that efficiently distribute radio resources when the resources demand exceeds the resource availability. As a result, it is inevitable that some users will exhibit a poorer user satisfaction performance, and the objective is to comparatively analyse the resources distribution efficiency among the different radio resource assignment policies.

The DCEAM proposal achieves the higher user satisfaction levels for the real-time video users at the expense of web and, especially, email services that are more poorly served. In fact, Table III shows that using DCEAM, on average, almost $90 \%$ of video transmissions received resources, and the number of assigned resources corresponds to that needed to achieve their minimum QoS level $\left(l_{i}\right)$. On the other hand, although web and email users also received the number of resources required to satisfy their minimum QoS level, only $38.1 \%$ and $6.8 \%$ of them are served in each distribution round respectively. This performance difference is due to the service priorities, and the fact that no limit on the waiting time was established for non real-time users. The observed DCEAM performance can be explained as follows. At the beginning of each distribution round, most users will have no radio resources allocated (except active video users), and therefore a utility value equal to zero. As a result, realtime users will first be served until they are assigned enough radio resources to achieve a non-zero utility value $\left(x_{i}^{k} \geq l_{i}\right)$. In this case, real-time video users are rapidly served, resulting in a low waiting time and therefore a low percentage of unsent video frames. The low number of available radio resources per cell, the emulated high system load, and the established service priorities explain the high waiting time experienced by email users (see Fig. 4). The emulated conditions also explain why users tend to be served with the resources equivalent to their minimum QoS requests, resulting in low user throughputs and increased transmission durations. The consequent high transmission durations result in a high number of competing users at each distribution round, which also explains the observed channel access stagnation for low priority users. Low user throughputs also cause the measured percentage of video frames that need to be aborted (Table III). As previously explained, user satisfaction is a subjective concept, and it is interesting to observe the performance that can be achieved when relaxing the QoS demands. While the web service achieves a $53 \%$ user satisfaction level when considering the $3 \mathrm{GPP}$ QoS definition (i.e., satisfactory transmission in less than 4 seconds), this

TABLE I

USER SATISFACTION (\% OF USERS) WITH STATIC SCHEME

\begin{tabular}{cccc}
\hline \hline & Min QoS demand & Mean QoS demand Max QoS demand \\
\hline Email & 66.05 & 77.22 & 70.59 \\
Web & 79.38 & 79.99 & 76.32 \\
16kbps video & 74.88 & 81.11 & 43.42 \\
32kbps video & 57.17 & 38.22 & 23.20 \\
64kbps video & 25.93 & 9.93 & 22.11 \\
\hline \hline
\end{tabular}

TABLE II

USER SATISFACTION (\% OF USERS) WITH PROPOSED SCHEMES

\begin{tabular}{ccccccc}
\hline \hline & DCEAM & DCELM & MAUI & RATE & $\begin{array}{c}\text { Modified } \\
\text { DCEAM }\end{array}$ & $\begin{array}{c}\text { Adapted } \\
\text { DCELM }\end{array}$ \\
\hline Email & 13.42 & 15.09 & 91.12 & 10.09 & 44.27 & 14.9 \\
Web & 53.02 & 57.48 & 45.01 & 46.38 & 60.31 & 53.2 \\
16kbps video & 86.42 & 63.71 & 99.94 & 89.91 & 70.91 & 86.25 \\
32kbps video & 87.43 & 79.64 & 81.79 & 88.54 & 71.08 & 87.41 \\
64kbps video & 86.81 & 83.65 & 85.48 & 85.15 & 70.45 & 87.23 \\
\hline \hline
\end{tabular}


TABLE III

BANKRUPTCY RADIO RESOURCE ASSIGNMENTS

\begin{tabular}{|c|c|c|c|c|c|c|c|c|c|}
\hline & \multicolumn{3}{|c|}{ DCEAM } & \multicolumn{3}{|c|}{ DCELM } & \multicolumn{3}{|c|}{ Adapted DCELM } \\
\hline & $\begin{array}{l}\text { Assigned } \\
\text { resources } \\
\text { per round }\end{array}$ & $\begin{array}{c}\text { Abort/Unsen } \\
\text { video frames } \\
(\%)\end{array}$ & $\begin{array}{c}\text { Average number/\% } \\
\text { of served } \\
\text { users per round }\end{array}$ & $\begin{array}{l}\text { Assigned } \\
\text { resources } \\
\text { per round }\end{array}$ & $\begin{array}{c}\text { Abort/Unsent } \\
\text { video frames } \\
(\%)\end{array}$ & $\begin{array}{c}\text { Average number/\% } \\
\text { of served users per } \\
\text { round }\end{array}$ & $\begin{array}{l}\text { Assigned } \\
\text { resources } \\
\text { per round }\end{array}$ & $\begin{array}{c}\text { Abort/Unsent } \\
\text { video frames } \\
(\%)\end{array}$ & $\begin{array}{c}\text { Average number/\% } \\
\text { of served users per } \\
\text { round }\end{array}$ \\
\hline Email & 1.16 & - & $0.21 / 6.79$ & 1.11 & - & $0.24 / 7.76$ & 1.13 & - & $0.22 / 6.78$ \\
\hline Web & 1.86 & - & $1.17 / 38.12$ & 1.34 & - & $1.89 / 67.50$ & 1.87 & - & $1.17 / 38.46$ \\
\hline $16 \mathrm{kbps}$ video & 1.09 & $10.51 / 3.07$ & $0.76 / 86.53$ & 1.04 & $13.60 / 22.69$ & $0.61 / 56.55$ & 1.05 & $10.77 / 2.98$ & $0.77 / 86.86$ \\
\hline $32 \mathrm{kbps}$ video & 2.03 & $11.15 / 1.44$ & $0.80 / 90.90$ & 1.62 & $19.88 / 0.49$ & $0.94 / 96.91$ & 2.01 & $11.19 / 1.40$ & $0.78 / 91.11$ \\
\hline $64 \mathrm{kbps}$ video & 3.71 & $13.08 / 0.12$ & $0.87 / 97.36$ & 3.31 & $16.33 / 0.02$ & $0.93 / 99.68$ & 3.72 & $12.65 / 0.12$ & $0.86 / 97.41$ \\
\hline
\end{tabular}

value can be increased to $63 \%$ and $70 \%$ when relaxing the QoS constraint to 5 and 6 seconds respectively. However, for email services, even if the QoS satisfaction criterion is relaxed, the percentage of satisfactory transmissions for the lowest priority service hardly improves due to the high waiting times. To overcome the DCEAM performance limitation for non-priority users, a modified DCEAM approach considering user waiting times has also been implemented. The modified DCEAM's operation is similar to the original DCEAM, but modifies the order at which users with equal utility values are served (i.e., it modifies $<_{L}$ ). While in this case DCEAM will assign the resources to the higher priority services, the modified DCEAM approach assigns them to the users experiencing the higher waiting times. The results depicted in Table II and Fig. 4 highlight that considering users waiting times helps increasing service fairness at the expense of reducing the QoS levels for the most demanding services, although they still maintain homogeneous user satisfaction levels as targeted with the bankruptcy proposals.

Tables II and III also depict the performance achieved with the DCELM proposal. The obtained results indicate that the original DCELM implementation significantly degrades the real-time user performance compared to DCEAM, while only slightly improving the less priorised services. This is due to the fact that the DCELM proposal initially assigns to all users the resources corresponding to their maximum demand $\left(c_{i}\right)$, and then unassigns resources to users experiencing the higher utility levels. As a result, users with higher possibilities to maintain radio resources are those that achieve lower utility values with these resources. Moreover, when a radio resource is unassigned to users that were allocated the number of radio resources corresponding to their minimum QoS threshold (users maintain $l_{i}$-1 assigned resources), these users achieve a utility value equal to zero. Following the DCELM principle, these users will not be unassigned the remaining resources (if they still have) until the rest of users experience a null utility value. Consequently, users requiring only one radio resource (users with $l_{i}=1$ ) will be those that first loose the assigned resources, which partly explains the poor performance for email and $16 \mathrm{kbps}$ video users. Table III also shows that DCELM results in that users requiring more than one resource to achieve their minimum QoS level (users with $l_{i}>1$ ) are generally assigned resources below this minimum threshold. Consequently, the initial DCELM implementation can result in a resource allocation not meeting the minimum QoS threshold. Such non-optimal resource allocation results in lower DCELM performance compared to the DCEAM implementation that always seeks to guarantee the minimum QoS level. Fig. 5 also shows that the resource assignments obtained with DCEAM correspond to higher utility values per traffic class, and that in fact such values are very close to the utility values corresponding to the minimum QoS level defined in Fig. 1. On the other hand, DCELM assignments result in very low utility values for service classes that require more than one resource to achieve
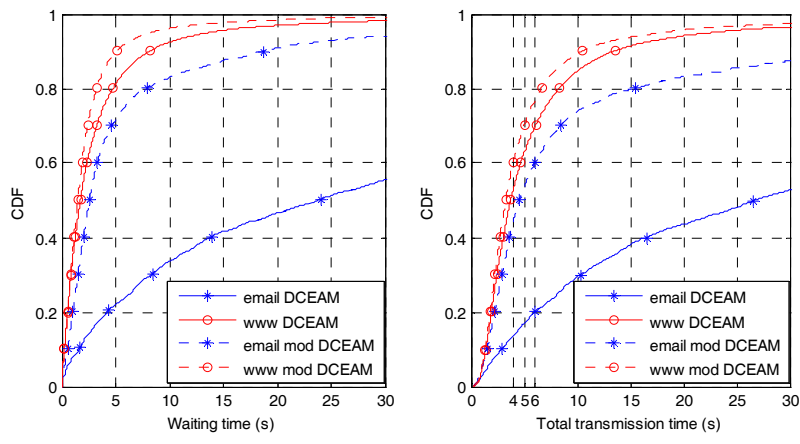

Fig. 4. CDF of waiting and total transmission times for email and web.

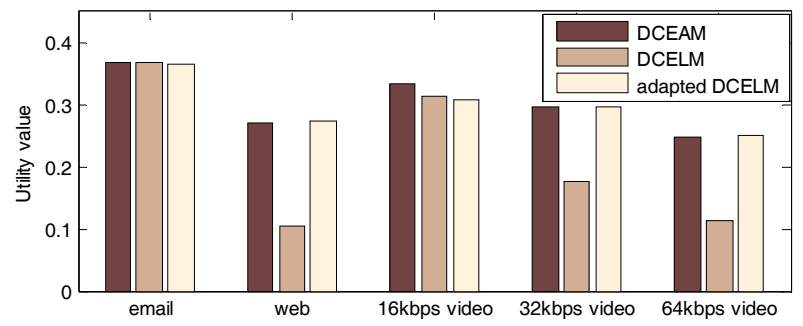

Fig. 5. Assigned utility values. 
their minimum QoS levels.

To overcome the initial limitations of DCELM, a modified approach has also been implemented. This approach follows the original DCELM operation except that when a radio resource must be unassigned to a user experiencing its minimum utility value (a resource must be unassigned to a user with $l_{i}$ assigned resources resulting in a utility value equal to zero), instead of only unassigning one resource, all allocated resources are unassigned and distributed among the other users. Table II and Fig. 5 show that with the adapted DCELM proposal, a similar performance to that achieved with DCEAM can be obtained. The performance of both proposals has also been evaluated in lower load scenarios (Fig. 6). The obtained results show that both techniques maintain their similar user satisfaction levels under varying operating conditions. However, for an equal user satisfaction, DCEAM improves the throughput (or reduces the transmission delay) for some services compared to adapted DCELM (see Fig. $7^{3}$ ). This is the case because of the different resource assignments resulting from the DCEAM and adapted DCELM techniques.

MAUI's performance is also reported in Tables II and V. The obtained results show that MAUI can significantly improve the overall system performance. Only web users do not achieve high user satisfaction levels, although a relaxed QoS satisfaction definition could significantly improve the web performance statistics given that $62.09 \%$ and $72.33 \%$ of web pages could be transmitted in less than 6 and 8 seconds respectively using MAUI. As observed in Table IV, MAUI tends to allocate a very high number of resources to fewer simultaneous active users. Although this might result in instantaneous lower resources fairness, what is important is not that resources are fairly distributed but that the QoS users perceive is fairly distributed. By assigning a significant amount of resources to a single user, MAUI increases the user's throughput and reduces its transmission time, which allows quickly freeing the resources and burstly serving a new user. In fact, while an average of 8.82 and 8.86 users are demanding resources in each distribution round when DCEAM and adapted DCELM are applied respectively, only 5.21 users on average participate in a distribution round when MAUI is applied. Despite improving the overall performance compared to the bankruptcy proposals for the reference simulated scenario, DCEAM and DCELM provide some of the most demanding services with

${ }^{3}$ For clarity, the figure only shows the traffic services for which a difference between both resource assignment policies has been observed.

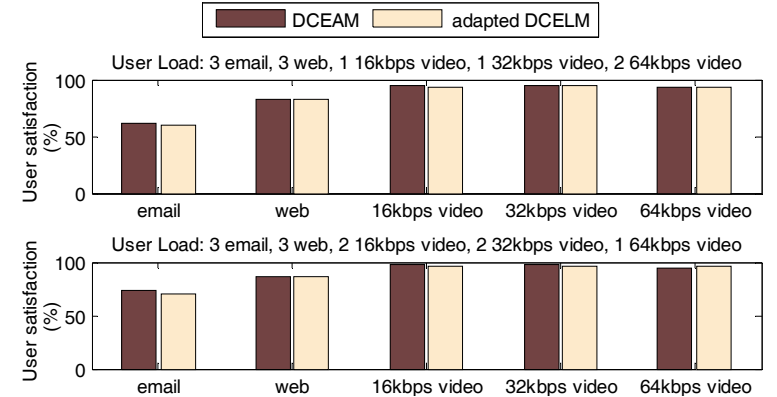

Fig. 6. User satisfaction levels under low load scenarios.
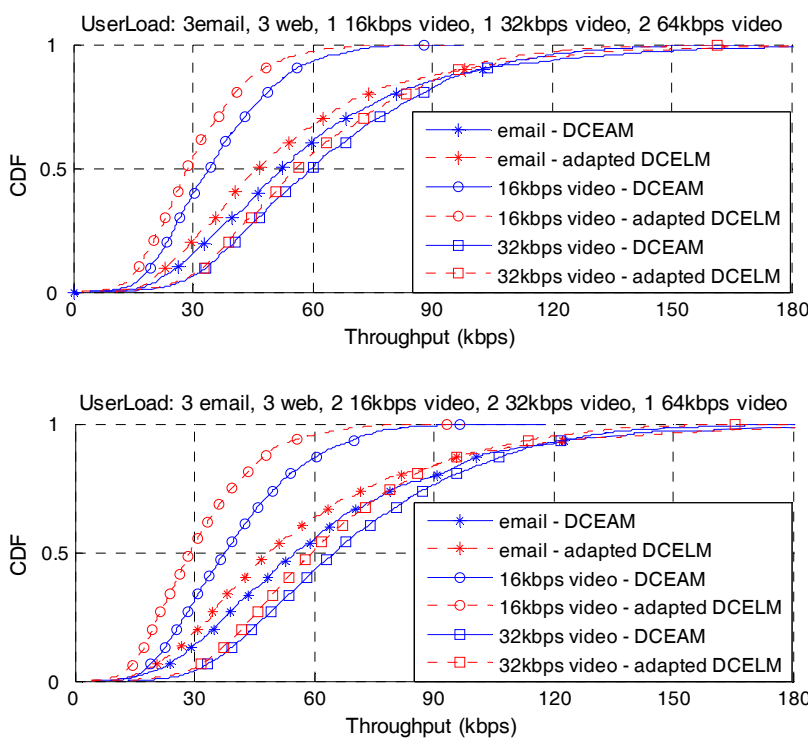

Fig. 7. Measured throughput under low load scenarios.

TABLE IV

MAUI AND RATE OPERATION

\begin{tabular}{c|ccc|ccc}
\hline \hline & \multicolumn{3}{|c|}{ MAUI } & \multicolumn{3}{c}{ RATE } \\
\hline & $\begin{array}{c}\text { Assigned } \\
\text { channels } \\
\text { per } \\
\text { round }\end{array}$ & $\begin{array}{c}\text { Abort/ } \\
\text { Unsent } \\
\text { video } \\
\text { frames } \\
(\%)\end{array}$ & $\begin{array}{c}\text { Average } \\
\text { number/\% } \\
\text { of served } \\
\text { users per } \\
\text { round }\end{array}$ & $\begin{array}{c}\text { Assigned } \\
\text { channels } \\
\text { per } \\
\text { round }\end{array}$ & $\begin{array}{c}\text { Abort/ } \\
\text { Unsent } \\
\text { video } \\
\text { frames } \\
(\%)\end{array}$ & $\begin{array}{c}\text { Average } \\
\text { number/\% } \\
\text { of served } \\
\text { users per } \\
\text { round }\end{array}$ \\
\hline Email & 6.65 & - & $0.10 / 99.99$ & 1.43 & - & $0.19 / 5.99$ \\
Web & 7.99 & - & $0.16 / 4.85$ & 1.18 & - & $1.59 / 49.15$ \\
$16 \mathrm{kbps}$ video & 4.91 & $0.06 / 0.01$ & $0.39 / 99.95$ & 1.17 & $6.76 / 3.33$ & $0.75 / 89.04$ \\
32kbps video & 7.39 & $2.24 / 15.980 .27 / 40.51$ & 2.01 & $10.18 / 1.28$ & $0.84 / 94.24$ \\
64kbps video & 7.45 & $1.98 / 12.550 .39 / 57.50$ & 3.35 & $14.68 / 0.17$ & $0.95 / 98.42$ \\
\hline \hline
\end{tabular}

higher satisfaction levels. In addition, the bankruptcy based proposals better adapt and operate under different and more capacity constrained scenarios. Fig. 8 represents the obtained user satisfaction levels for a higher system load. It is interesting to note that when the load increases, the bankruptcy-based proposals obtain a higher system performance, and are the only techniques capable to provide homogeneous user satisfaction levels for the more demanding services (under the simulated conditions, user satisfaction fairness can only achieved for real-time video users due to the shortage of radio 
resources). This desirable characteristic has been shown to be independent of the system conditions for the bankruptcy-based proposals, but not for the other simulated techniques.

Tables II and IV also depict the results achieved with the RATE mechanism, which is the only proposal that does not base its decision criterion on the defined utility functions, but rather on the estimated required data rates to achieve satisfactory user transmissions. Interestingly, the bankruptcy and RATE proposals achieve similar results indicating that the established utility functions adequately reflect the user needs. The data summarized in Table V shows that for low user loads, both RRM techniques achieve very similar QoS performance for all services. However, when the user load increases, RATE achieves a lower user satisfaction level for the real-time video service with higher bit rate. For example, while DCEAM obtained user satisfaction levels of $78.69 \%$ and $72.86 \%$ for cell loads of 18 and 19 users per cell, RATE's performance decreased to $72.53 \%$ and $63.41 \%$. It is important to highlight that the user satisfaction

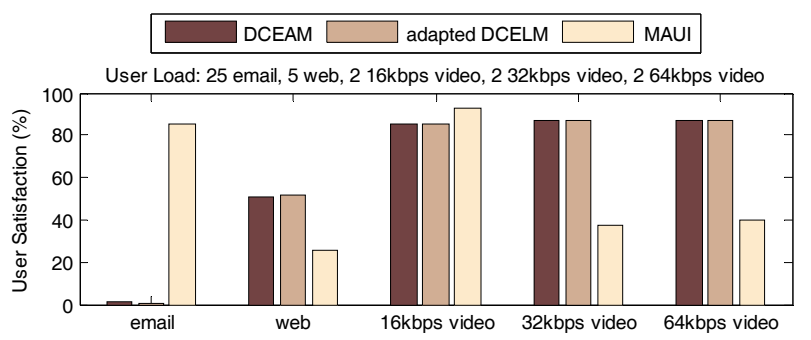

Fig. 8. User satisfaction under high system loads.

TABLE V

DCEAM AND RATE USER SATISFACTION (\% OF USERS) FOR DIFFERENT LOADS

\begin{tabular}{|c|c|c|c|c|c|c|}
\hline & \multicolumn{2}{|c|}{$\begin{array}{l}3 \text { email, } 3 \text { web, } \\
116 \mathrm{kbps} \text { video, } \\
132 \mathrm{kbps} \text { video, } \\
264 \mathrm{kbps} \text { video }\end{array}$} & \multicolumn{2}{|c|}{$\begin{array}{l}5 \text { email, } 5 \text { web, } \\
216 \mathrm{kbps} \text { video, } \\
332 \mathrm{kbps} \text { video, } \\
364 \mathrm{kbps} \text { video }\end{array}$} & \multicolumn{2}{|c|}{$\begin{array}{l}5 \text { email, } 5 \text { web, } \\
216 \mathrm{kbps} \text { video, } \\
332 \mathrm{kbps} \text { video, } \\
464 \mathrm{kbps} \text { video }\end{array}$} \\
\hline & DCEAM & RATE & DCEAM & RATE & DCEAM & RATE \\
\hline Email & 83.23 & 81.47 & 17.65 & 18.84 & 10.00 & 10.97 \\
\hline Web & 61.91 & 62.64 & 3.76 & 3.06 & 0.86 & 0.00 \\
\hline $16 \mathrm{kbps}$ video & 95.33 & 96.92 & 63.81 & 65.93 & 43.67 & 44.47 \\
\hline $32 \mathrm{kbps}$ video & 94.49 & 95.50 & 72.39 & 73.20 & 56.27 & 58.49 \\
\hline $64 \mathrm{kbps}$ video & 93.79 & 92.46 & 78.69 & 72.53 & 72.86 & 63.41 \\
\hline
\end{tabular}

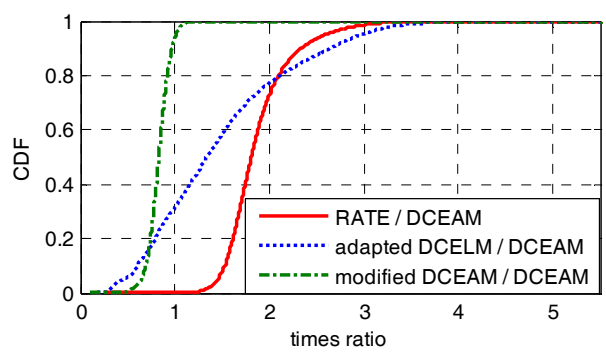

Fig. 9. CDF of the computational cost relation between techniques. levels obtained for the other services are very similar for both techniques. In addition, it is important to emphasize that RATE needs to compute in real-time, and for each distribution round, the user required data rates, while the DCEAM and DCELM distribution processes are only based on utility functions defined offline.

The computational cost of the different radio resource assignment policies is illustrated in Fig. 9, where the ratio between the time needed by RATE and DCEAM to distribute the radio resources per distribution round is represented. Fig. 9 shows that there is a zero probability that the ratio between the times needed by RATE and DCEAM to distribute their resources is lower than one, which means that RATE always requires more time to distribute the radio resources than DCEAM. It is also interesting to note that in $25 \%$ of the simulated distribution rounds, the time spent by RATE is more than double the time required by DCEAM. It is then interesting to analyse which bankruptcy based proposal would require the lower computational cost. To this aim, Fig. 9 also represents the simulation time relation between different radio resource assignment techniques. First, it is important to highlight that the modified DCEAM proposal required less time than the original DCEAM to achieve a solution, and that the mean ratio between the times required by both techniques is 0.84 . Despite achieving similar performance, the results depicted in Fig. 9 show that in $70 \%$ of the simulated distribution rounds, adapted DCELM required more time to find a solution than DCEAM. The results in Fig. 9 also show that in about $25 \%$ of the distribution rounds, adapted DCELM required more than double the time DCEAM needed to distribute resources among competing users; the other way around was only observed in $5 \%$ of the distribution rounds. To provide an indication of the absolute computational cost of each radio resource assignment technique, Fig. 10 shows the $\mathrm{CDF}$ of the simulation time needed by each technique to distribute the radio resources among demanding users ${ }^{4}$. It is interesting to note that while the DCEAM proposals needed a maximum of $30 \mathrm{~ms}$ for all their distribution rounds, this value increased to $80 \mathrm{~ms}$ for RATE and adapted DCELM.

To analyse the relation between simulation time and number of users in each distribution round, Fig. 11 depicts the percentage of distribution rounds that required more time to end their radio resources distribution process with DCEAM and adapted DCELM respectively as a function of the number of active users.

\footnotetext{
${ }^{4}$ The simulations were conducted with a $3 \mathrm{GHz}$ Xeon Dual Core processor with 4 GB of RAM.
} 


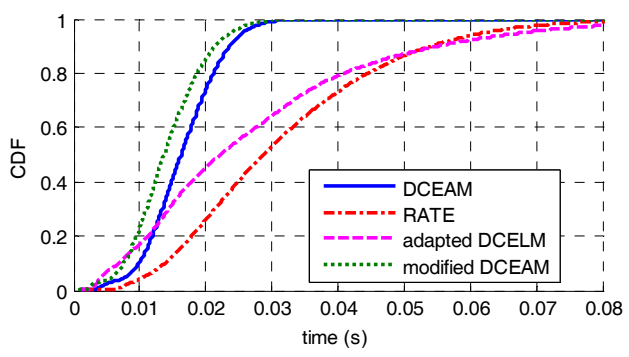

Fig. 10. CDF of the simulation time needed to end a radio resource distribution round among active users.

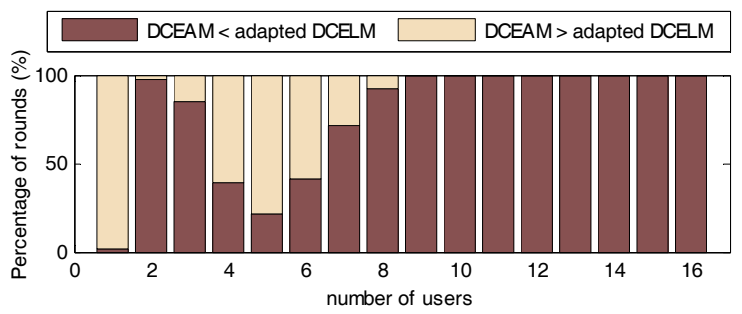

Fig. 11. Percentage of distribution rounds that required more time with DCEAM and adapted DCELM as a function of the number of active users.

This figure shows that when the number of users participating in the distribution process is higher than 6 , adapted DCELM requires more time to distribute the resources in most of the distributions rounds. This trend is always observed when the number of active users is higher than 8 . These results clearly highlight the better computational behaviour of the DCEAM proposal as the system load increases. In addition, it is important to emphasize that the distribution of users among service classes also impacts the computational cost. Fig. 12 depicts the percentage of occasions in which DCEAM had a higher computational cost than adapted DCELM and vice versa. When a higher percentage of the users participating in the distribution process correspond to the most demanding service, i.e., the $64 \mathrm{kbps}$ video, adapted DCELM spends more time to achieve the distribution solution. On the other hand, DCEAM requires more computational time when a higher percentage of the users demands email and web services.

\section{CONCLUSION}

This work has proposed the application of bankruptcy theory to design efficient and fair radio resource assignment techniques in multimedia mobile radio networks. To this aim, several bankruptcy rules have been adapted to operate with resources of discrete nature. The proposed bankruptcy techniques are based on previously established utility functions that try to characterise the different QoS requirements per traffic type. The obtained results have demonstrated the potential of bankruptcy techniques to address radio

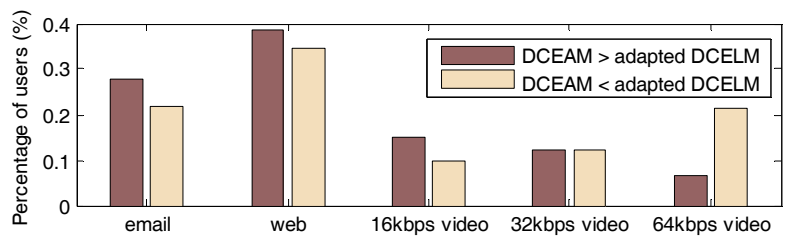

Fig. 12. Distribution of users among service types per distribution round when considering 6 active users.

resource management problems, in particular thanks to their capability to guarantee uniform user satisfaction levels through a proper definition of the utility functions. Contrary to what has been observed with other mechanisms, this property has been shown to be independent of the system load. In addition, this paper has also investigated the computational cost of bankruptcy-based techniques, and its dependence with the system load and user distribution per service class. The obtained results have shown that the proposed bankruptcy-based policies have a low computational cost compared to other assignment policies.

\section{ACKNOWLEDGEMENTS}

This work has been supported by the Ministry of Education and Science (Spain) and FEDER funds under the project TEC2005-08211-C02-02, MTM2008-06778c02-01 and TEC2008-06728, and by the Ministry of Industry, Tourism and Trade under the CELTIC project CP5-013/TSI-02400-2008-113.

\section{REFERENCES}

[1] Vivier E., Terré M., Fino B. Determination of all possible resource allocations in a packet communication network. In Proc. 56th IEEE Vehicular Technology Conference, Vancouver, Canada, Sept. 2002; 4, 2346-2350.

[2] Singh J. Performance Aspects of Enhanced Radio Resource Management with QoS Differentiation in UMTS Network. In Proc. 6th IEEE International Conference on $3 G$ and Beyond, London, UK, Nov. 2005; 1-5.

[3] Al-Manthari B., Nasser N., Hassanein H. Downlink Scheduling With Economic Considerations for Future Wireless Networks. IEEE Transactions on Vehicular Technology 2009; 58: 824-835.

[4] Vlacheas, P. T., Kolokotroni, E. A., Tragos, E. Z. and Theologou, M. E., A hybrid multirate MAC protocol providing trade-off between throughput and fairness in future TD-CDMA networks. European Transactions on Telecommunications 2010, 21(1): 64-72.

[5] Schmeink, A., On fair rate adaption in interferencelimited systems. European Transactions on Telecommunications 2011; Article first published online, Apr 2011.

[6] Pei, X., Zhu, G., Wang, Q., Qu, D. and Liu, J., Economic model-based radio resource management with QoS 
guarantees in the CDMA uplink. European Transactions on Telecommunications (2010), 21(2): 178-186.

[7] Dramitinos M., Stamoulis G. D., Courcoubetis C. An auction mechanism for allocating the bandwidth of networks to their users. Computer Networks 2007; 51(18): 4979-4996.

[8] Malla A., El-Kadi M., Olariu S., Todorova P. A Fair Resource Allocation Protocol for Multimedia Wireless Networks. IEEE Transactions on Parallel and Distributed Systems 2003; 14: 63-71.

[9] Thomson W. Axiomatic and game-theoretic analysis of bankruptcy and taxation problems: a survey. Mathematical Social Sciences 2003; 45: 249-297.

[10]Lucas-Estañ M.C., Gozalvez J., Sanchez-Soriano J., Pulido M., Calabuig D. Multi-Channel Radio Resource Distribution Policies in Heterogeneous Traffic Scenarios. In Proc. IEEE $66^{\text {th }}$ Vehicular Technology Conference (VTC2007-Fall), Baltimore, USA, Sept. 2007, 16741678.

[11] Gozalvez J., Lucas-Estañ M.C., Sanchez-Soriano J. User QoS-based Multi-Channel Assignment Schemes under Multimedia Traffic Conditions. In Proc 4th International Symposium on Wireless Communications Systems (ISWCS2007), Trondheim, Norway, Oct. 2007; 113-117.

[12]Barford P., Crovella M. Generating representative web workloads for network and server performance evaluation. In Proc. Int'l Conf. Measurement and Modeling of Computer Systems (SIGMETRICS/PERFORMANCE 1998), New York, USA, June 1998, 151-160.

[13]Ho J., Zhu Y., Madhavapeddy S. Throughput and buffer analysis for GSM general packet radio service (GPRS). In Proc. IEEE Wireless Comms. and Networking Conf. (WCNC 1999), New Orleans, LA, Sept. 1999, 1427-1431.

[14] Lázaro O., Girma D., Dunlop J. H.263 video traffic modelling for low bit rate wireless communications. In Proc. IEEE Personal, Indoor and Mobile Radio Communications (PIMRC 2004), Barcelona, Spain, Sept. 2004; 2124-2128.

[15]Hanzo L., Cherriman P., Streit J. Wireless video communications: second to third generation systems and beyond. IEEE Press, 2001.

[16] Herrero C., Martinez R. Balanced Allocation Methods for Claims Problems with Indivisibilities. CORE Discussion Paper 2006/66, July 2006. Available at SSRN: http://ssrn.com/abstract $=944416$.

[17] López-Benítez M., Lucas-Estañ M., Gozalvez J. A dynamic radio simulation platform for the study of radio resource management techniques in heterogeneous wireless systems. In Proc. 9th ACM/IEEE International Symposium on Modeling, Analysis and Simulation of Wireless and Mobile Systems, Málaga, Spain, Oct. 2006.

[18] Gozalvez J., López-Benítez M., Lázaro O. Link adaptation algorithm for improved wireless transmission of delay-sensitive packet data services. Electronic Letters 2005; 41(14): 813-815.

\section{AUTHORS’ BIOGRAPHIES}

M.C. Lucas-Estañ received a Telecommunications Engineering degree in 2007 and a DEA in industrial and telecommunications technologies in 2009, both from the University Miguel Hernandez of Elche in Spain. In 2007, she joined the Uwicore research laboratory where she is conducting her $\mathrm{PhD}$ on joint radio resource management policies in multimedia heterogeneous wireless systems. She received an Orange Foundation award at the 2007 Spanish Young Scientist Contest organized by the Spanish Ministry of Education for her research on RRM for wireless networks. She is currently contributing to the mHop project.

J. Gozalvez received an electronics engineering degree from the Engineering School ENSEIRB (Bordeaux, France), and a $\mathrm{PhD}$ in mobile communications from the University of Strathclyde, Glasgow, U.K. Since October 2002, he is with the University Miguel Hernandez of Elche, Spain, where he is currently an Associate Professor and Director of the Uwicore Laboratory. At Uwicore, he is leading research activities in the areas of wireless vehicular communications, radio resource management, and heterogeneous wireless systems. He currently serves as Mobile Radio Senior Editor of IEEE Vehicular Technology Magazine. He is TPC Co-Chair of the 2011 IEEE VTC-Fall, and was TPC Co-Chair of the 2009 IEEE VTC-Spring, and General Co-Chair of the 3rd ISWCS 2006. He is also the founder and General Co-Chair of IEEE $\mathrm{WiVeC}$ in 2007, 2008, and 2010. He has been elected to the Board of Governors of the IEEE Vehicular Technology Society (2011-2013) and to the IEEE Distinguished Lecturers program of the IEEE Vehicular Technology Society.

J. Sanchez-Soriano received a Mathematics Science Degree in 1991 and a PhD in Mathematics Science in 1998 both from Murcia University (Spain). He was Director of the Operations Research Center of the University Miguel Hernández (Spain). $\mathrm{He}$ is Professor in the Department of Statistics, Mathematics and Computer Science of the University Miguel Hernández. $\mathrm{He}$ is author or co-author of more than 60 research articles published in journals and books. His previous and current research interests include operations research and game theory and their applications, in particular to radio resource management, logistic problems and market design. 